\title{
Lidar-based road terrain recognition for passenger vehicles
}

\section{Shifeng Wang*}

National Demonstration Center for Experimental Opto-Electronic

Engineering Education,

School of Opto-Electronic Engineering,

Changchun University of Science and Technology,

Changchun, 130022, China

Email: SF.Wang@cust.edu.cn

${ }^{*}$ Corresponding author

\section{Sarath Kodagoda and Lei Shi}

Centre for Autonomous Systems,

University of Technology Sydney,

Ultimo 2007, Australia

Email: Sarath.Kodagoda@uts.edu.au

Email: Lei.Shi-1@uts.edu.au

\section{Ning Xu}

School of Opto-Electronic Engineering, Changchun University of Science and Technology,

Changchun 130022, China

Email: NingXu1995@163.com

\begin{abstract}
The road terrain type is important information about a passenger vehicle's surroundings. It suggests an appropriate control algorithm and driving strategy. In this paper, a Lidar sensor is employed to reconstruct the road surface and extract features for terrain classification. The experiment vehicle was driven on four specific road terrains at a variety of speeds. The speed dependency and the effect of using principal component analysis were investigated. The simulation experimental results show that this Lidar sensorbased approach is feasible and robust for passenger vehicles in a range of outdoor scenarios.
\end{abstract}

Keywords: terrain recognition; feature extraction; classification; machine learning; passenger vehicles.

Reference to this paper should be made as follows: Wang, S., Kodagoda, S., Shi, L. and $\mathrm{Xu}$, N. (2017) 'Lidar-based road terrain recognition for passenger vehicles', Int. J. Vehicle Design, Vol. 74, No. 2, pp.153-165. 
Biographical notes: Shifeng Wang received his $\mathrm{PhD}$ at the University of Technology Sydney, Australia in 2013 and is currently an Associate Professor at School of Opto-Electronic Engineering, Changchun University of Science and Technology, China. His research interests include environment perception for land vehicles, opto-electronic detection devices and machine learning.

Sarath Kodagoda received his $\mathrm{PhD}$ at Nanyang Technological University, Singapore in 2004 and is currently an Associate Professor at the University of Technology Sydney, 2007, Australia. His research interests include robotics, human robot interaction and driver assistance systems. He is Member of IEEE Robotics and Automation Society and IEEE Communications Society.

Lei Shi received his $\mathrm{PhD}$ at the University of Technology Sydney, Australia in 2013 and is currently a Research Associate at the University of Technology Sydney, 2007, Australia. His research interests are machine learning, spatial statistics, sensor and signal processing.

Ning $\mathrm{Xu}$ is currently an undergraduate student of Changchun University of Science and Technology, China. His research interest is opto-electronics detection and cloud point data process.

This paper is a revised and expanded version of a paper entitled 'Road terrain type classification based on laser measurement system data' presented at Australasian Conference on Robotics and Automation, Wellington, New Zealand, 3-5 December, 2012.

\section{Introduction and related work}

For the environment perception of passenger vehicles, knowledge about the road terrain is part of the important information the vehicles should acquire. Different road terrains can have a significant impact on vehicle handling, ride, quality and stability (Bing, 2010). A method of road terrain classification using Lidar sensor is presented in this paper.

A Lidar sensor was used by Nishiwaki et al. (2012) to solve the terrain perception problem. The robot was able to navigate on unrehearsed terrain without having any prior knowledge or assumptions of the road. With the usage of the Lidar sensor, the terrain was reconstructed in a large-scale map for the robot to plan paths. Lidar has also been applied for outdoor rover. Montemerlo et al. (2004) concentrated on identifying the terrain by dividing view fields into patches with different sizes. This method used a terrain model which arose from the limited spatial resolution of the point cloud. This approach presented successfully identified obstacles on the terrain of the mobile robot environment. Andersen et al. (2006) did not divide the terrain map into segments. The terrain was classified to find a traversable region through scans. Seven distinct characteristics were fused to identify passable roads and obstacles which allowed the robot to navigate autonomously.

Lidar sensor is used not only on low-speed small-sized robots but also on a highspeed road vehicle. Urmson et al. (2004) concentrated on a road vehicle application which navigated on unknown terrain at high speed. However, owing to the vibration of the vehicle while moving, the forward Lidar was particularly affected by mechanical excitation in the pitch direction. A terrain measurement method was proposed in which 
the calibration surface was compared with the absolute reference road profile (Smith and Ferris, 2010). Another way to estimate the road surface is a Doppler effect-based approach to derivate the torsional slip, friction and other parameters (Holton and Ahmadian, 2008).

An accelerometer was mounted on a vehicle to estimate the road surface (Ward and Iagnemma, 2009). The road terrain recognition system was supposed to be independent of the vehicle's speed. The spatial frequency features were extracted from the road profile for the terrain classification. However, further research (Wang, 2012) discovered that the sampling limit to the road surface and non-ideal nature characteristics resulted in speed dependency.

A method for road terrain classification presented in this paper is a Lidar data-based approach. Lidar sensor can provide range values with high spatial density sampling points, which contributes to reconstruct the road surface, and high sampling frequency, which helps to overcome the effect of the changing speed of the vehicle and obtain higher and more reliable classification rates.

As shown in Figure 1, CRUISE (CAS Research UTE for Intelligence, Safety and Exploration), a Ford Courier utility vehicle, is our experiment platform. It is equipped with a Lidar that scans the road surface, four wheel encoders that are used to obtain the vehicle's speed, a laptop in the cab for data collection operating connecting with a PC104 computer installed in the back tray for Lidar data logging, and a bank of batteries to provide the required power for all the equipment.

Figure 1 Research platform: CRUISE (see online version for colours)

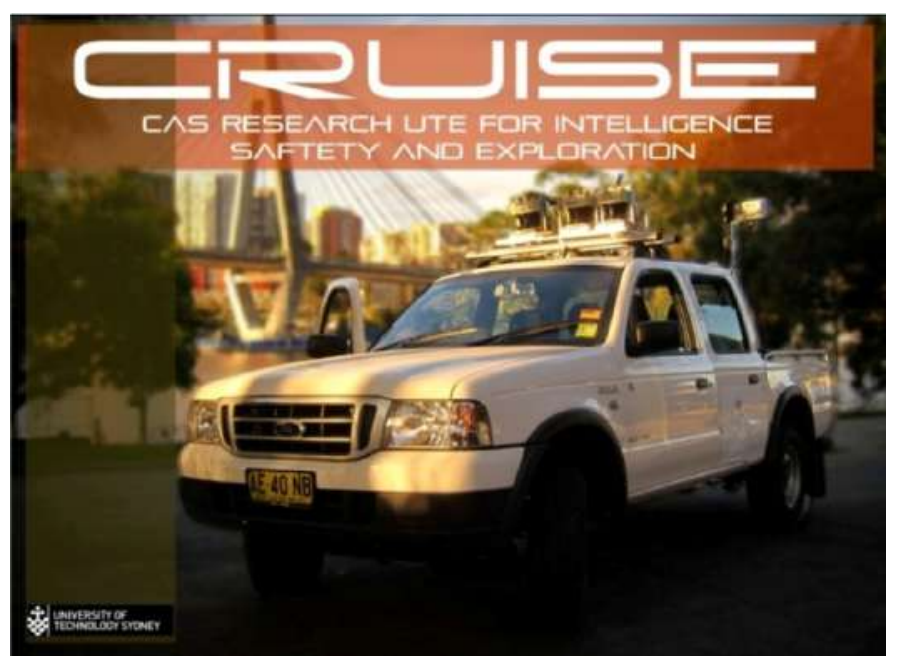

The following content is organised as follows: Section 2 describes the methodology of the road surface estimation, the feature extraction, the form of the feature matrix and the classifier selection; Section 3 introduces the experiment platform, data collection and the experiments; Section 4 presents the experiment results and their analysis; and Section 5 gives the conclusion and suggests future work of this research. 


\section{Methodology}

\subsection{Geometric arrangement of the Lidar sensor}

As shown in Figure 2, the Lidar sensor is mounted on a supporting frame of CRUISE looking-downward to the ground in the $z$-direction. The laser beam scans the road terrain along the $x$-axis at $50 \mathrm{~Hz}$ with $270^{\circ}$ field of view. Each scan reflects 541 range values with $0.5^{\circ}$ angular resolution. When CRUISE moves along the $y$-axis, the Lidar data leaves a trace of three-dimensional (3D) point cloud of the road surface.

Figure 2 The geometric arrangement of the Lidar (see online version for colours)

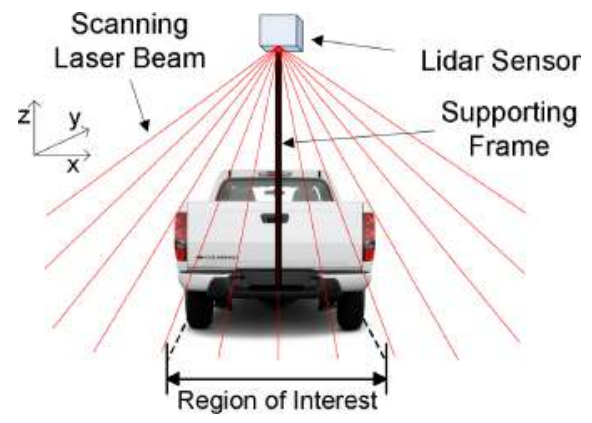

\subsection{Speed sampling}

The speed of the vehicle is estimated by tapping the Hall effect sensors that are used for the ABS (antilock brake system). The sine-like signals are isolated and amplified, then processed by a microcontroller to act as speed data. Hence, the speed of the vehicle can be obtained as

$$
v(t)=\frac{\pi D_{w} f_{v}}{4 p} \sum_{i=1}^{4} n_{i}
$$

where $D_{w}$ is the external diameter of the tyre; $f_{v}$ is the sampling frequency, $10 \mathrm{~Hz}$, which depends on how often the microcontroller transfers counting data to the computer; $p$ is the number of teeth around the wheel hub, which in this case is 44 teeth; $i$ is the index of the four wheels; and $n_{i}$ is a number from each wheel, which indicates how many teeth have passed by in $0.1 \mathrm{~s}$.

\subsection{Displacement calculation}

The vehicle displacement is estimated as

$$
x(t)=\int_{0}^{t}|v(t)| \mathrm{d} t
$$

where $v(t)$ is the measured vehicle's speed that is obtained by (1). Since the sampling frequency is $50 \mathrm{~Hz}$ for the Lidar sensor and $10 \mathrm{~Hz}$ for the speed, a nearest-neighbour interpolation is applied to $x(t)$ data to make certain each vertical displacement $y_{i}$ has a corresponding horizontal displacement data $x_{i}$. 


\subsection{Road terrain surface sampling}

As can be seen in Figure 3, the vertical coordinate, $z_{i}$, of each range measurement is easily able to be obtained by

$$
z_{i}=H-r_{i} \cos \left(\theta_{i}\right)
$$

where, $r_{i}$ is the value of Lidar range, $\theta_{i}$ is the included angle from $z$-axis to current laser beam, and $H$ is the reference height from a relatively flat floor to the position of the Lidar sensor. In a similar way, the $x$-axis coordinate of the sampled points can be calculated as

$$
x_{i}=r_{i} \sin \left(\theta_{i}\right)
$$

The laser beam scans vertically down to the ground in a $270^{\circ}$ field of view, which covers road terrain that the vehicle has passed over and other objects on the road. Therefore, as shown in Figure 2, the region of interest is then narrowed down to $1.3 \mathrm{~m}$ which is actually the width of the vehicle for the terrain recognition purpose. This brings the distance between two sampling points of a scan to $\sim 2 \mathrm{~cm}$ on the road surface. Besides, this Lidar is mounted $\sim 2.2 \mathrm{~m}$ high above the road surface.

Figure 3 Road terrain surface calculations (see online version for colours)

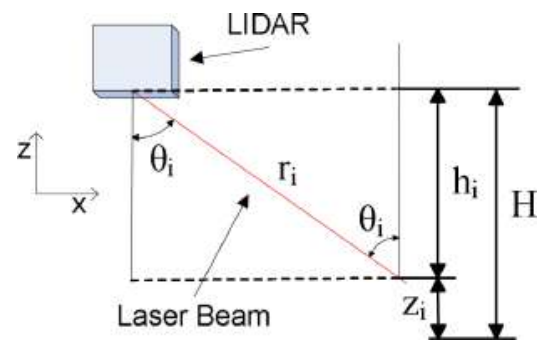

\subsection{Road terrain surface reconstruction}

As shown in Figure 4, the road terrain surface is estimated from the raw laser range data and the raw speed data from the wheel encoders.

Figure 4 Reconstruction procedure of the road terrain surface

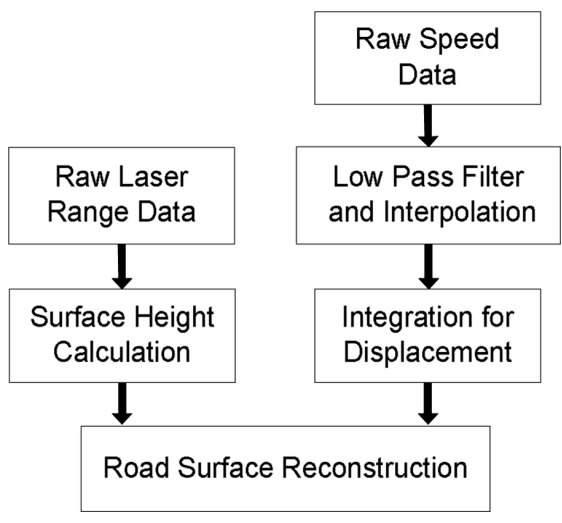


The raw laser range data is in a polar coordinate system which needs to be converted into rectangular coordinate system. A 3D view of the road surface can be generated after the procedure to estimate the longitudinal and lateral displacements of the point cloud data from the Lidar sensor. Such displacement profiles of different road terrains are shown in Figure 5. The irregularities and diversities of the surfaces suggest the possibility of qualitative assessment purely by visual inspection. It can be surmised that different vegetated terrain produces different surfaces apart from the above four types (Figure 5). Different terrain surfaces definitely present exclusive geometric parameters which will be described by the power spectral density (PSD) features (Welch, 1967).

Figure 5 The four reconstructed road terrain surfaces: (a) asphalt terrain; (b) concrete terrain; (c) grass terrain and (d) gravel terrain (see online version for colours)

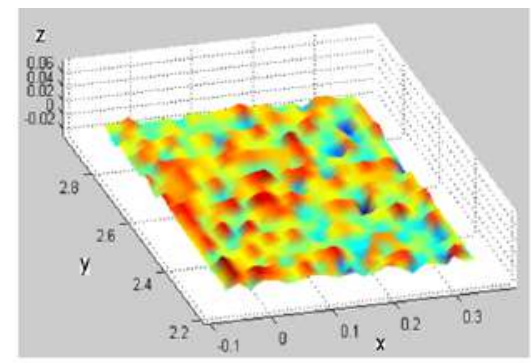

(a)

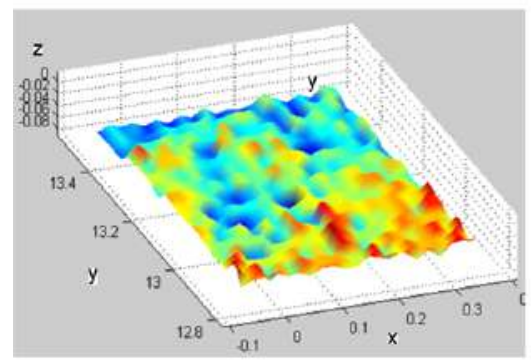

(c)

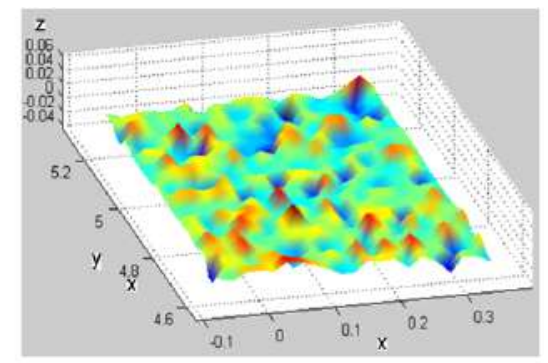

(b)

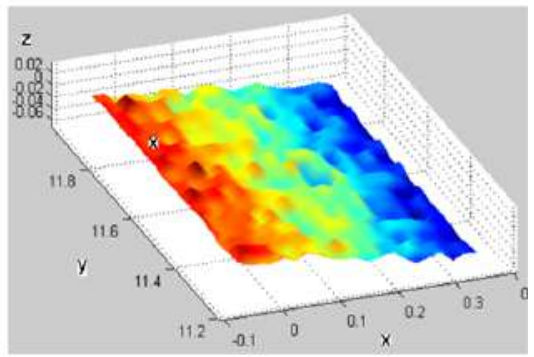

(d)

\subsection{Feature matrix}

The reconstructed 3D road surface point cloud has two directions for the feature extraction, as demonstrated in Figure 2, $y$-axis and $x$-axis. Owing to the variability of the speed, the resolution along the CRUISE moving direction ( $y$-axis) has more dissimilarity compared with the scanning direction ( $x$-axis) of the laser beam. In order to make the varying speed have a minimal effect to the recognition rate, the lateral components $(x$-axis) are employed for the feature extraction. Each scan is then carried out Fast Fourier Transformation (FFT) to form the PSD feature matrix using the method of Welch (1967).

$$
F=\left[\begin{array}{ccc}
F_{s_{1}, f_{1}} & \ldots & F_{s_{n}, f_{1}} \\
\vdots & \ddots & \vdots \\
F_{s_{1}, f_{m}} & \ldots & F_{s_{n}, f_{m}}
\end{array}\right] .
$$


In the feature matrix, as seen in equation (5), each column refers to a feature vector in which each element is a calculated value representing the PSD of a sample (a group of scans). Furthermore, from index 1 to $m$ indicate PSD value from lowest frequency component to highest one, while $n$ indicates a sample's sequence. For these elements computing, the start frequency, the step frequency, and the end frequency are decided as $0,0.1$, and $35 \mathrm{~Hz}$ through many prior tests. Specifically, for the first sample $(n=1)$, the first PSD value summarised from 0 to $0.1 \mathrm{~Hz}(m=1)$ is the element of $F_{s_{1}, f_{1}}$. Likely, $F_{s_{1}, f_{2}}$ presents the PSD value summarised from $0.1 \mathrm{~Hz}$ to $0.2 \mathrm{~Hz}$ of the first sample, then $F_{s_{n}, f_{m}}$ presents the PSD value summarised from $34.9 \mathrm{~Hz}$ to $35 \mathrm{~Hz}$ of the $n$th sample. Therefore, a feature matrix has $n$ samples and each sample contains $m$ features. Before sending to the classifier, this feature matrix needs to be normalised to reflect each row as $[0,1]$ and then be labelled manually to divide it into training dataset and testing dataset.

\subsection{Classifier selected}

Neural network classifier and naïve Bayes classifier, and support vector machines (SVM) classifiers were selected for evaluation to find out the most appropriate classifier in terms of implementation time and accuracy rate for this case.

To get more robust and reliable classification results, a method for selecting training and testing dataset, the $K$-fold cross validation, is then employed (Picard, 1984). This method divides available data into $K$-folds. This procedure then will implement $K$ times unless all the data of each fold is processed as testing data. An average value of these $K$ classification results is then calculated as the final accuracy of the classifier. As illustrated in Picard and Cook (1984), set $K$ as 5 is an optimised value that always leads to a stabilised result. In this classifier selection test, the parameter is set as five-fold cross validation to assess the mean performance of the whole methodology using parts of the dataset.

The comparison off-line tests were carried out via the tool kit WEKA (Bouckaert et al., 2010). The SVM classifier was then selected for the following experiments because of its faster computing speed and higher accuracy.

\section{Experiment}

\subsection{Experimental platform}

CRUISE is utilised for the experiments. As shown in Figure 6, the Lidar sensor is mounted upon the back tray of the vehicle scanning downward for sampling the road terrain. The mounting position is aligned with the central axis of CRUISE and the laser beam scanning plane is perpendicular to the ground. When the vehicle moves forward, the Lidar sensor scans the surface of the road and then leaves a structured point cloud corresponding to the road surface.

\subsection{Data collection}

CRUISE's driving routes on the four road types during the data collection experiment are shown in Figure 7. Continuous data logging ensued in elliptical roads with few other running vehicles. The grass road was more challenging to find. Having found only a 
$100 \mathrm{~m}$ stretch of a grass road, CRUISE then had to travel on this short road over several times with intensive vibration. Difficulties were also encountered in the search for a gravel road. A $1 \mathrm{~km}$ long gravel road was eventually found in the Royal National Park.

Figure 6 The mounted downward-scanning Lidar (see online version for colours)
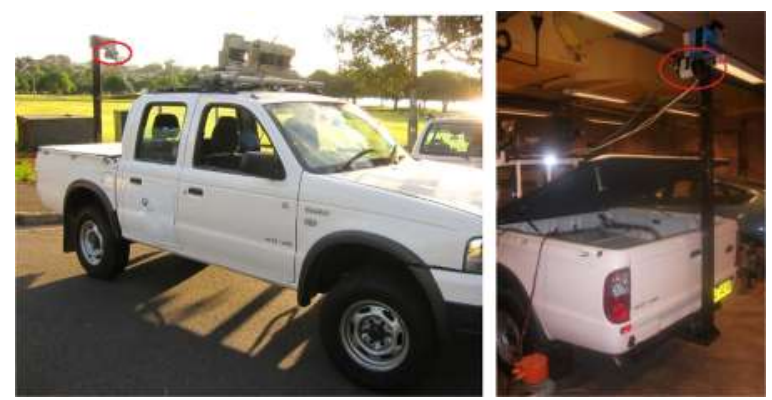

Figure 7 data collection routes of four road terrains: (a) the asphalt route; (b) the concrete route; (c) the grass route and (d) the gravel route (see online version for colours)

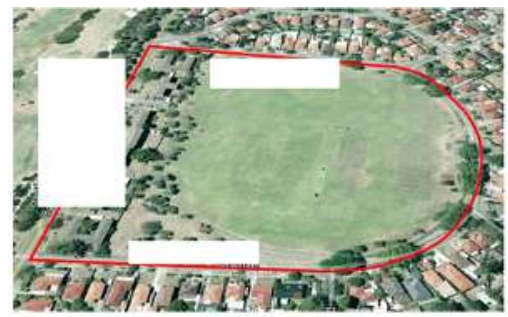

(a)

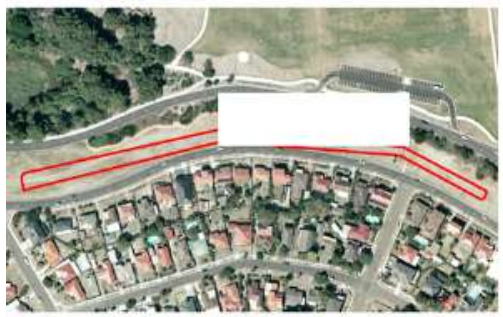

(c)

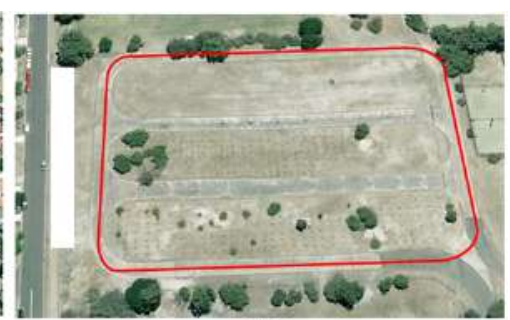

(b)

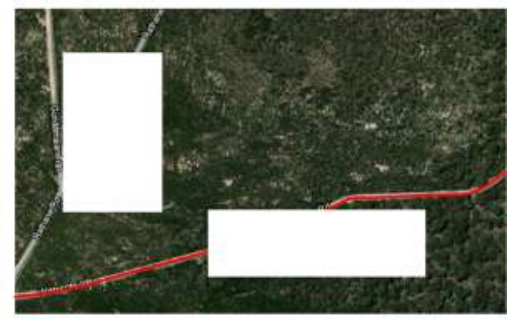

(d)

For the purpose of investigating speed independency, the CRUISE is driven on four types

of roads at different speeds. Considering driving safety and practical requirements, the data are collected on the asphalt and the concrete roads at speeds of 20,30 and $40 \mathrm{~km} / \mathrm{h}$; the grass road at speeds of $20 \mathrm{~km} / \mathrm{h}$ and $30 \mathrm{~km} / \mathrm{h}$; and the gravel road at a speed of $20 \mathrm{~km} / \mathrm{h}$, approximately. The driver and passengers felt critical vibration while CRUISE was running on the grass roads at speed of over $20 \mathrm{~km} / \mathrm{h}$, something neither comfortable nor safe for the driver or the vehicle with all the attached equipment.

Apart from the places described above, the experiment also involved more public roads. Since the speed and routes were not restricted, the driving circumstance contained many starts, stops and turns. Moreover, the driving routes have numbers of asphalt road types including tunnels, highways and suburb distributor roads. 


\section{Experiment results}

\subsection{Speed independency}

As for this point cloud-based experiments, the selected data with particular speeds is used to investigate the speed independency of the Lidar sensor, and trained classification model to do the testing for the road terrain classification. The actual labels are manually configured to compare with the results according to the visible road recognised by the operator during the experiment. The speed independency is investigated to prove the assumption that the Lidar data collected has been minimally impacted by the changing speed of the vehicle. This is reasonable, because comparing the possible speed of the vehicle, the Lidar sensor performs relatively quick sampling which captures range data in a few microseconds.

This hypothesis is then tested using asphalt terrain data and gravel terrain data with a range of different speeds. The formed feature matrix with labels is split and selected by the particular speeds for the training stage, and then selected for the testing purpose with different speeds from the training dataset, separately.

Table 1 shows the classification results as the CRUISE is running at some specific speeds. For instance, the first row shows that the training dataset is asphalt and gravel terrains both at $20 \mathrm{~km} / \mathrm{h}$ while the testing dataset is asphalt terrain at $40 \mathrm{~km} / \mathrm{h}$ and gravel terrain at $10 \mathrm{~km} / \mathrm{h}$, and the asphalt terrain is correctly classified by $100.0 \%$ while the gravel terrain is $99.2 \%$. The rest of the table shows some other classification results that stem from different combinations of training and testing dataset with different speeds. Obviously, these extremely high classification rates lead to the conclusion that the Lidar data-based approach is speed independent.

Table 1 Speed independency experiment

\begin{tabular}{llcc}
\hline Training dataset $(\mathrm{km} / \mathrm{h})$ & Testing dataset $(\mathrm{km} / \mathrm{h})$ & $\begin{array}{c}\text { Asphalt accuracy } \\
(\%)\end{array}$ & $\begin{array}{c}\text { Gravel accuracy } \\
(\%)\end{array}$ \\
\hline Asphalt@20 \& Gravel@20 & Asphalt@40 \& Gravel@10 & 100.0 & 99.2 \\
Asphalt@20 \& Gravel@20 & Asphalt@30 \& Gravel@30 & 100.0 & 100.0 \\
Asphalt@20\&Gravel@20 & Asphalt@30 \& Gravel@10 & 100.0 & 99.2 \\
Asphalt@30 \& Gravel@30 & Asphalt@20 \& Gravel@10 & 100.0 & 100.0 \\
Asphalt@30 \& Gravel@30 & Asphalt@40 \& Gravel@20 & 100.0 & 100.0 \\
\hline
\end{tabular}

As mentioned, the acceleration data-based approach has the shortcoming of speed dependency (Wang et al., 2012). It suggests a sufficiently large training dataset that should cover as many speeds as possible for better performance. However, as observed through this experiment, when using Lidar data, it is not essential to collect the data with all possible speeds for the training stage but to do so still produces reasonable high classification accuracies.

\subsection{Simulation experiment}

As shown in Table 2, Lidar data with limited speeds are selected to train while other Lidar data are used to test. The classifier is set as SVM. The actual labels are manually set to compare with the testing results. As can be seen, the amount of the data that a Lidar 
sensor produces is quite large. The amounts of the training samples of all four classes are approximately equal so as to conduct balanced and appropriate training. The quite large testing dataset is arranged as the vehicle runs on four types of terrain randomly. It needs to be clarified that this testing dataset has not been used for any training which tries to simulate a real-world circumstance. It can be noted the asphalt road is the most widely available type whereas the concrete and the grass roads are very hard to find for data collection in Sydney, Australia.

Table 2 Simulation experiment dataset

\begin{tabular}{lcccccc}
\hline & \multicolumn{3}{c}{ Training dataset } & \multicolumn{3}{c}{ Testing dataset } \\
\cline { 2 - 7 } Road terrains & $\begin{array}{c}\text { Speed } \\
(\mathrm{km} / \mathrm{h})\end{array}$ & $\begin{array}{c}\text { Displacement } \\
(\mathrm{m})\end{array}$ & $\begin{array}{c}\text { Number of } \\
\text { scans }\end{array}$ & $\begin{array}{c}\text { Speed } \\
(\mathrm{km} / \mathrm{h})\end{array}$ & $\begin{array}{c}\text { Displacement } \\
(\mathrm{m})\end{array}$ & $\begin{array}{c}\text { Number of } \\
\text { scans }\end{array}$ \\
\hline Asphalt & $20,30,40,50$ & 328 & 4005 & $0 \sim 70$ & 3948 & 61,194 \\
Concrete & 20,30 & 284 & 3950 & $0 \sim 45$ & 952 & 11,836 \\
Grass & 10 & 304 & 5383 & $0 \sim 25$ & 876 & 11,386 \\
Gravel & 20,30 & 312 & 4225 & $0 \sim 35$ & 2712 & 24,208 \\
Total & & 1228 & 17,563 & & 8488 & 108,624 \\
\hline
\end{tabular}

Figure $8(a)-(d)$ show the label sequences of the simulation experiment. The horizontal axis is the index of laser beam scans. There are four points (1, 2, 3 and 4) on the vertical axis that show the class labels. The predicted labels classified are marked by green circles while the actual labels are represented by blue lines. The details are arranged to simulate that the vehicle runs from one type of terrain to another by random segments of road shown in Figure 8. Taking a qualitative look at Figure 8(a)-(d), it can be seen that each of the classes is classified fairly accurately, save for a few exceptions, where concrete terrain is confused to be grass terrain and grass terrain is wrongly labelled as concrete terrain. This Lidar data-based approach is capable of distinguishing between these four types of terrains.

As can be seen in Table 3, in the experiment, the classification accuracies of asphalt, concrete, grass and gravel road terrains are $87.9 \%, 72.8,83.5 \%$ and $85.1 \%$, respectively. In addition, this experiment is without the principal component analysis (PCA; Jolliffe, 2002) process. Generally, though the asphalt and concrete terrain are intuitively hard to be distinguished from each other, they are classified reasonably well. Moreover, it can be seen that some small segments of concrete road are recognised as grass roads $17.8 \%$ of the time. This is mainly due to the structures of concrete and grass roads, which are somehow so similar that they confuse the classifier. Overall, the approach presented in this paper is feasible, specifically, with average classification accuracy $82.3 \%$.

The classification results shown in Table 4 are with the PCA process applied to the feature matrices which leads to relatively lower results when comparing with Table 3. Although the accuracies of concrete and gravel terrains classification rates are slightly higher, the asphalt one significantly falls down extremely, as well as the average classification accuracy. Hence, these results indicate that the PCA process to the feature matrix does not help in increasing the classification rate using Lidar data for this task. 
Table 3 The statistics of the simulation without PCA process

\begin{tabular}{lcccc}
\hline Testing & $\begin{array}{c}\text { Tsphaitt@20,30, } \\
40,50(\mathrm{~km} / \mathrm{h})\end{array}$ & $\begin{array}{c}\text { Concrete @,20,30 } \\
(\mathrm{km} / \mathrm{h})\end{array}$ & $\begin{array}{c}\text { Grass@10 } \\
(\mathrm{km} / \mathrm{h})\end{array}$ & $\begin{array}{c}\text { Gravel@,20,30 } \\
(\mathrm{km} / \mathrm{h})\end{array}$ \\
\hline Asphalt@0 70 & $87.9 \%$ & $0.5 \%$ & $0.1 \%$ & $11.5 \%$ \\
Concrete@0 45 & $0.0 \%$ & $72.8 \%$ & $17.8 \%$ & $9.4 \%$ \\
Grass@0 25 & $0.0 \%$ & $14.2 \%$ & $83.5 \%$ & $2.3 \%$ \\
Grave1@0 35 & $8.5 \%$ & $4.5 \%$ & $1.9 \%$ & $85.1 \%$ \\
\hline
\end{tabular}

Average classification accuracy: $82.3 \%$.

Figure 8 (a) Lidar based experiment label sequence: segment 1; (b) Lidar based experiment label sequence: segment 2; (c) Lidar based experiment label sequence: segment 3 and

(d) Lidar based experiment label sequence: segment 4 (see online version for colours)

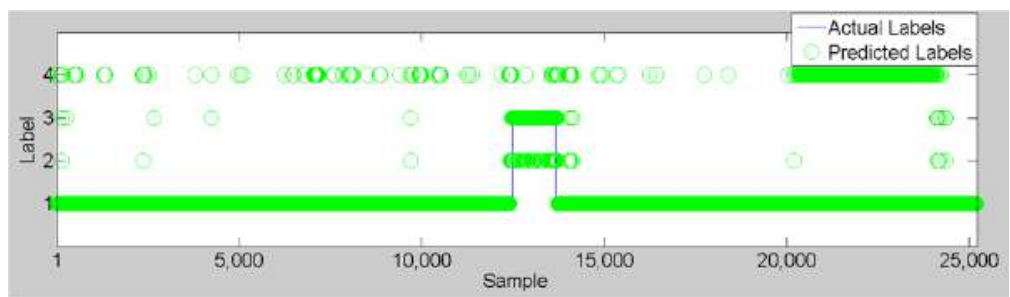

(a)

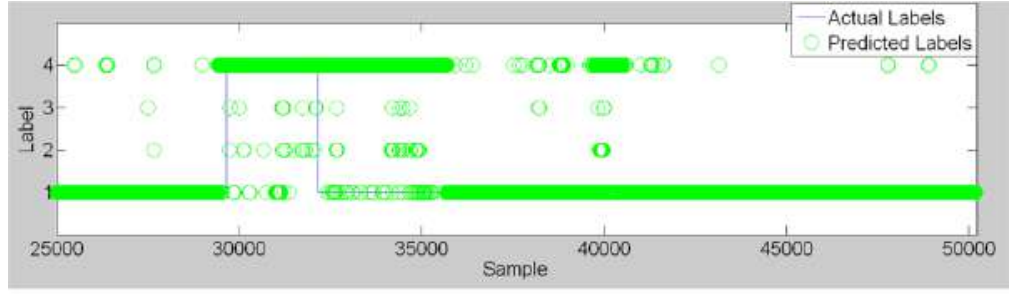

(b)

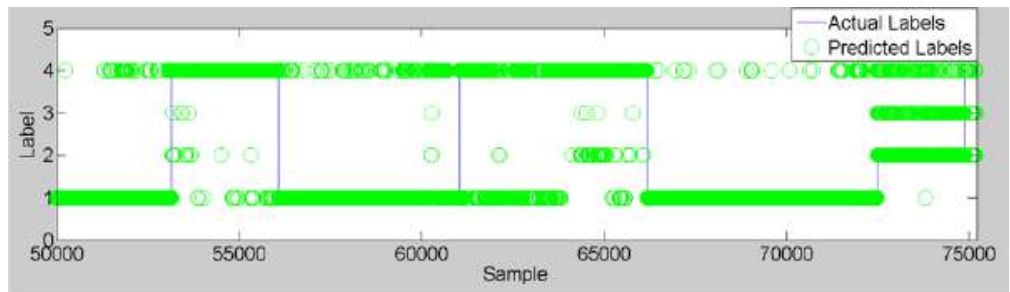

(c)

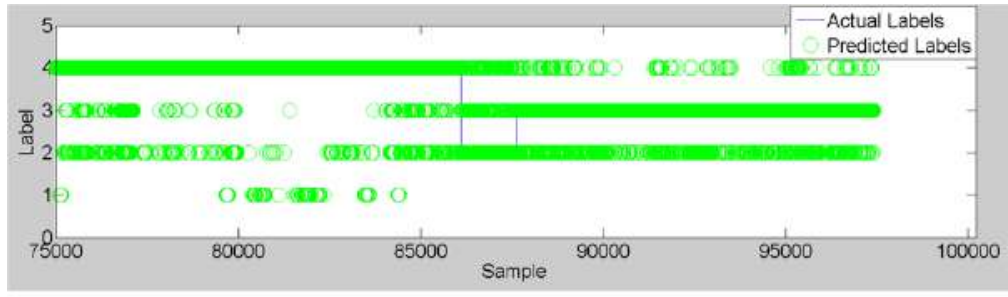

(d) 
Table 4 The experimental results using downward Lidar data with PCA process

\begin{tabular}{lcccc}
\hline Testing & $\begin{array}{c}\text { Trainingalt@20,30, } \\
40,50(\mathrm{~km} / \mathrm{h})\end{array}$ & $\begin{array}{c}\text { Concrete@20,30 } \\
(\mathrm{km} / \mathrm{h})\end{array}$ & $\begin{array}{c}\text { Grass @ 10 } \\
(\mathrm{km} / \mathrm{h})\end{array}$ & $\begin{array}{c}\text { Gravel@,20,30 } \\
(\mathrm{km} / \mathrm{h})\end{array}$ \\
\hline Asphalt@0 70 & $21.2 \%$ & $38.3 \%$ & $16.5 \%$ & $24.0 \%$ \\
Concrete@0 45 & $0.0 \%$ & $79.1 \%$ & $20.9 \%$ & $0.0 \%$ \\
Grass@0 25 & $0.0 \%$ & $19.5 \%$ & $76.7 \%$ & $3.8 \%$ \\
Gravel@0 35 & $0.0 \%$ & $3.1 \%$ & $7.6 \%$ & $89.3 \%$ \\
\hline
\end{tabular}

Average classification accuracy: $69.8 \%$.

\section{Conclusion}

In this paper, the approach to classify road terrain types using Lidar data was presented. The employed Lidar sensor scanned the road surface that the vehicle travelled on. With the collected speed of the vehicle, the road surface can be reconstructed using the 3D point cloud data from the Lidar sensor. The PSD features were extracted to form the feature matrix for the classification. The speed independency was investigated to verify the assumption that Lidar had much less speed effect. The simulation experimental results showed that it was a feasible method to classify road terrain types using Lidar sensor. Furthermore, the usage of PCA presented lower classification rates. It should be noted that the range value data from the Lidar can be affected by the vibration when the vehicle is moving on higher roughness road. Owing to this distance varying between the Lidar sensor and the road surface, the PSD features could be less accurate which could lead to lower classification rate. Mounting an inertial measurement unit (IMU) on the Lidar can improve this situation which corrects the small displacement of the Lidar.

However, the downward Lidar employed in this approach scans the road terrain surface that the vehicle has already passed over. This means the testing results of the terrain types are kind of afterward reports. It would be more significant to know the road terrain types that the vehicle has not passed over but are ahead of the vehicle. Therefore, the future work is considered to put another Lidar sensor on the roof of the CRUISE that looks forward onto the upcoming road. It is expected to predict the road terrain types that the vehicle is about to run onto. In addition more types of terrain are considered to be involved to make this approach more widely applicable.

\section{Acknowledgements}

This work is funded by National Natural Science Foundation of Jilin Province (20150101047JC), Changchun University of Science and Technology for Young Scholar (XQNJJ-2014-05), Funding of Science and Technology Commission of Shanghai Municipality (13dz2260100), National Key Basic Research Program of China (2014CB744200) and Open Projects of Shanghai Key Laboratory of Deep Space Exploration Technology (DS201509-002, DS201507-001). This work is also supported by the Centre for Autonomous Systems of the University of Technology Sydney. 


\section{References}

Andersen, J.C., Blas, M.R., Ravn, O., Andersen, N.A. and Blanke, M. (2006) 'Traversable terrain classification for outdoor autonomous robots using single 2D laser', Integrated ComputerAided Engineering, Vol. 13, pp.223-232.

Bing, Z. (2010) 'Ride comfort research of off-road vehicle based on soft terrain', IEEE 11th Int. Conf. on Computer-Aided Industrial Design and Conceptual Design (CAIDCD), 17-19 November, Yiwu, China, pp.579-584.

Bouckaert, R.R., Frank, E., Hall, M.A., Holmes, G., Pfahringer, B., Reutemann, P. and Witten, I.H. (2010) 'WEKA-experiences with a java open-source project', J. Machine Learning Research, Vol. 11, pp.2533-2541.

Holton, C. and Ahmadian, M. (2008) Doppler Sensor for the Derivation of Torsional Slip, Friction and Related Parameters, Patent Application Number US20070765215, 12 June.

Jolliffe, I. (2002) Principal Component Analysis, 2nd ed., Springer-Verlag, New York.

Montemerlo, M. and Thrun, S. (2004) 'Muti-resolution pyramid for outdoor robot terrain perception', 19th Natioanl Conf. on Artificial Intelligence, San Jose, USA, pp.464-469.

Nishiwaki, K., Chestnutt, J. and Kagami, S. (2012) 'Autonomous navigation of a humanoid robot over unknown rough terrain using a laser range sensor', Int. J. Robotics Research, Vol. 31, Vol. 11, pp.1251-1262.

Picard, R.R. and Cook, R.D. (1984) 'Cross validation of regression models', J. the American Statistical Association, Vol. 387, No. 79, pp.575-583.

Smith, H. and Ferris, J.B. (2010) 'Calibration surface design and validation for terrain measurement systems', Journal of Testing and Evaluation, Vol. 38, No. 4, pp.1-8.

Urmson, C., Anhalt, J., Clark, M., Galatali, T., Gonzalez, J.P., Gowdy, J., Gutierrez, A., Harbaugh, S., Johnson-Roberson, M., Kato, H., Koon, P., Peterson, K., Smith, B., Spiker, S., Tryzelaar, E. and Whittaker, W. (2004) High Speed Navigation of Unrehearsed Terrain: Red Team Technology for Grand Challenge 2004,Tech. Rep. CMU-RI-TR-04-37), Robotics Institute, Carnegie Mellon University, Pettsburgh, PA.

Wang, S., Kodagoda, S. and Khushaba, R. (2012) 'Towards speed-independent road-type classification', Int. Conf. on Control, Automation, Robotics and Vision (ICARCV), Guangzhou, China, pp.614-619.

Ward, C.C. and Iagnemma, K. (2009) 'Speed-independent vibration-based terrain classification for passenger vehicles', Vehicle System Dynamics, Vol. 47, No. 9, pp.1095-1113.

Welch, P.D. (1967) 'The use of fast Fourier transform for the estimation of power spectra: a method based on time averaging over short, modified periodograms', IEEE Trans. Audio Electroacoust, Vol. 15, August, pp.70-73. 